Ann. Génét. Sél. anim., x969, 1 (4), 4I3-422.

\title{
FRÉQUENCES A L'ÉQUILIBRE DU GÈNE D'ORIGINE ALPINE \\ RESPONSABLE DE L'ABSENCE DE CORNES ET DE L'INTERSEXUALITÉ DANS DES POPULATIONS DE CHÈVRES
}

\author{
J.-J. LAUVERGNE \\ Station centrale de Génétique animale, \\ Centre national de Recherches zootechniques, 78 -Jouy-en-Josas \\ Institut national de la Recherche agronomique
}

RÉSUMÉ

Dans une population où l'on ne conserve que des reproducteurs caprins sans cornes porteurs homo- ou hétérozygotes du gène $P$, la fréquence gamétique femelle à l'équilibre est de 0,5 , la fréquence gamétique mâle à l'équilibre variant entre 0,521 et 0,661 selon l'intensité de la sélection qui est exercée sur les mâles $P P$. Les valeurs des fréquences gamétiques mâles observées en Allemagne et en Suisse $(0,5544$ et 0,6444 respectivement) se rangent dans cet intervalle. C'est une preuve de la validité des conceptions que l'on a sur le comportement du gène $P$ et de la précision de l'estimation des paramètres qui caractérisent son action. Dans un tel modèle, à chaque génération, il faut écarter de la reproduction 50 p. 100 des femelles et de 28 à 48 p. 100 des mâles.

\section{INTRODUCTION}

Dans des pays d'élevage caprin comme la Suisse ou l'Allemagne on s'est longtemps efforcé et l'on s'efforce encore d'obtenir des lignées où tous les animaux sont dépourvus de cornes. Pour cela, on écarte soigneusement de la reproduction, tous les animaux cornus. Or, dans ces populations, le mutant dominant responsable de la disparition des cornes est pléiotrope pour diverses anomalies du tractus génital entraînant la stérilité de certains animaux mottes homozygotes femelles ou mâles (LAUVERGNE, I969).

De ces actions sélectives en sens contraire, il résulte des états d'équilibre pour les fréquences géniques que nous nous proposons tout d'abord d'étudier théoriquement. En deuxième lieu, nous essaierons de vérifier si ce modèle est effectivement suivi. 


\section{I. - LE MODÈLE MATHÉMATIQUE}

Comme cela correspond d'ailleurs aux exigences des livres généalogiques des races sans cornes nous nous plaçons dans le cas où tous les reproducteurs sont sans cornes, c'est-à-dire où les coefficients de sélection des homozygotes sans cornes $p p$ sont nuls dans les deux sexes.

\section{A. - L'équation de passage de la fréquence gamétique.}

Nous avons vu (LAUVERGNE, I969) que la pénétrance $c$ de la stérilité chez les femelles $P P$ était totale, c'est dire que le coefficient de sélection de ce génotype est nul chez les chèvres. Comme nous venons de voir que toutes les chèvres cornues $p p$ étaient éliminées il ne restera, dans le sexe femelle, que des animaux hétérozygotes $P p$. La fréquence gamétique de $P$ chez les femelles sera donc constante et égale à 0,5 .

Chez les mâles, outre les animaux cornus $p p$ qui sont écartés de la reproduction par la sélection des éleveurs, un certain nombre de boucs homozygotes $P P(b)$ présentent des atteintes de sténose épididymaire partielles $\left(\beta_{1}\right)$ ou généralisées $\left(\beta_{2}\right)$ qui, dans ce dernier cas entraînent la stérilité des mâles. Outre cette élimination naturelle, l'homme peut intervenir à l'encontre des homozygotes $P P$ atteints de sténose partielle qu'un examen externe attentif peut déceler et qui sont alors, tout ou partie $(0<s<\mathrm{I})$, écartés de la reproduction.

Les forces de sélection que nous avons vues jusqu'à présent à l'ceuvre pour favoriser les hétérozygotes par rapport aux homozygotes $P P$ jouent aussi en sens contraire, on constate en effet que la prolificité des boucs $P P$ est supérieure à celle des boucs $P p$ ou $p p$ (RICORDEAU, I969). Le coefficient de surprolificité des $P P$ par rapport aux $P p$ étant désigné par $r$, le coefficient I- $S$ de sélection génotypique de $P P$ par rapport à $P p$ dans l'établissement de l'équation de passage de la fréquence gamétique chez les mâles se formule ainsi :

$$
\mathrm{I}-S=r\left[\mathrm{I}-b\left(s \beta_{1}+\beta_{2}\right)\right]
$$

Si l'on admet que $r, b, \beta_{1}$ et $\beta_{2}$ sont des constantes, $S$ est une fonction linéaire croissante de $s$ variant entre

$$
S_{0}=\mathrm{I}-r\left(\mathrm{I}-b_{2}\right) \text { et } S_{1}=\mathrm{I}-r(\mathrm{I}-b) \text { pour } 0<s<\mathrm{I}
$$

Les valeurs de $b$ et $\beta_{2}$ ont été calculées par LAUVERGNE (I969) à partir des données d'autres auteurs. Si l'on retient pour $b$ et $\beta_{2}$ les estimations établies à partir du travail de SchöNHERR (r954) qui est le plus complet et le plus détaillé sur la question ( $\left.b=0,9 \mathrm{I} 4, \beta_{2}=0,36 \mathrm{I}\right)$ et pour $r$ la valeur donnée par RICORDEAU (I969): $r=\mathrm{I}, 073$ l'intervalle de variation de $S$ est borné par les valeurs $S_{0}=0,28 \mathrm{I}$ (pour $s=$ o) et $\mathrm{S}_{1}=0,908$ (pour $s=\mathrm{I}$ ).

En désignant par $u_{n-1}$ et $u_{n}$ les fréquences de $P$ parmi les gamètes mâles utiles de, respectivement, la $n$-Ième et la nème génération l'équation de passage s'écrit :

$$
u_{n}=\frac{(\mathrm{I}-S) u_{n-1}+0,5}{(\mathrm{I}-S) u_{n-1}+\mathrm{I}}
$$




\section{B. - Recherche des valeurs d'équilibre, leur stabilité}

La formule (2) est l'équation d'une famille d'hyperboles équilatères. Les branches qui nous intéressent sont comprises dans le carré o, I du plan $u_{n-1} / u_{n}$ entre les courbes d'équation :

$$
u_{n}=\frac{0,7 \mathrm{I} 9 u_{n-1}+0,5}{0,7 \mathrm{I} 9 u_{n-1}+\mathrm{I}} \quad \text { et } \quad u_{n}=\frac{0,092 u_{n-1}+0,5}{0,092 u_{n-1}+\mathrm{I}}
$$

qui sont, dans ce domaine-là, croissantes à concavité tournée vers le bas (fig. I).

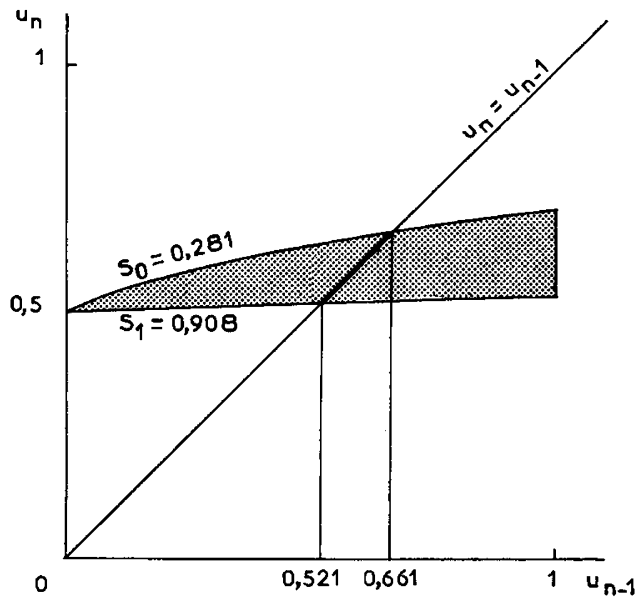

FIG. I. - Disposition de la famille des courbes $\mathrm{u}_{n}=\mathrm{f}\left(S_{1}, \mathrm{u}_{n-1}\right)$ dans le carré $\mathrm{O}, \mathrm{I}$ du plan $\mathrm{u}_{n-1} / \mathrm{u}_{n}$, $S$ variant entre $0,28 \mathrm{r}$ et 0,908 . En trait renforcé sur la première bissectrice le lieu d'intersection de ces courbes avec la première bissectrice dont les abscisses sont les valeurs d'équilibre de la fréquence gamétique mâle de $\mathbf{P}$.

Les valeurs d'équilibre de la fréquence génique sont les ordonnées du point d'intersection des courbes de la famille avec la première bissectrice, racines de 1'équation (2) dans laquelle on fait $u_{n}=u_{n-1}$ et qui s'écrit alors :

$$
(\mathrm{I}-S) u_{n}{ }_{n}+S u_{n}-0,5=0
$$

L'équation (3) n'admettant qu'une racine entre o et I pour l'intervalle de variation de $S$ considéré, il n'y a donc qu'une valeur d'équilibre $u_{e}$ qui s'écrit :

$$
u_{e}=\frac{-S+\sqrt{S^{2}+2(\mathrm{I}-S)}}{2(\mathrm{I}-S)}
$$

On déduit de la position continuellement croissante des courbes de la famille au voisinage de leur intersection avec la première bissectrice que l'équilibre est stable.

L'étude directe de $u_{e}$ fonction de $S$ dans l'équation (4) est moins simple que l'étude de la fonction inverse :

$$
S=\frac{0,5-u_{e}^{2}}{u_{e}\left(\mathrm{I}-u_{e}\right)}
$$


qui est continue croissante à concavité tournée vers le bas dans l'intervalle utile. On en déduit aisément que la fonction $u_{e}$ de $S$ est également continue décroissante à concavité orientée vers le bas dans l'intervalle de variation de $S$ défini ci-dessus. On a alors pour $u_{e}$ un intervalle de variation compris entre $u_{e}\left(S_{1}\right)=0,52 \mathrm{I}$ (tous les animaux atteints partiellement sont gardés) et $u_{e}\left(S_{0}\right)=0,66$ I (tous les animaux atteints partiellement sont écartés de la reproduction) (cf. fig. 2).

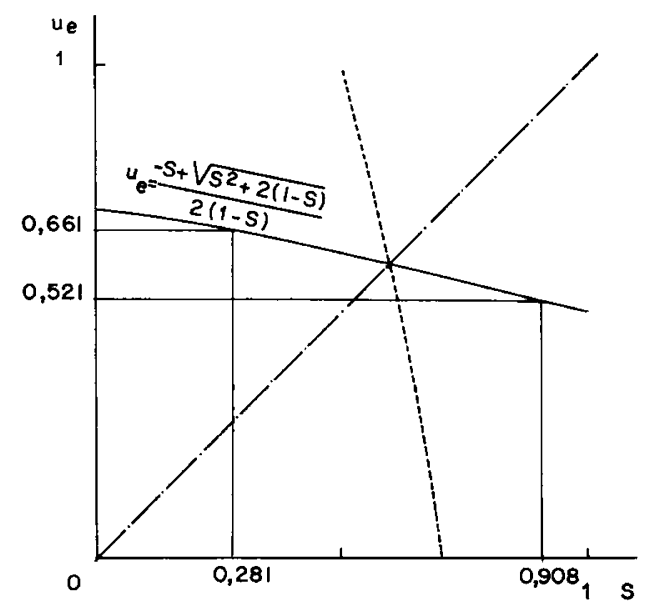

FIc. 2. - Fréquence gamétique $\mathrm{u}_{\mathbf{e}}$ de $\mathrm{P}$ chez les mâles fonction de la proportion $\mathrm{S}$ des máles de génotypes $\mathrm{PP}$ écartées de la reproduction pour anomalies épididymaires (en pointillé, la courbe inverse).

\section{C. - Vitesse d'obtention de l'équilibre}

On peut remarquer tout d'abord que, quelle que soit $S$ et la valeur de départ $u_{0}$, dès la première génération on a $u_{1}=0,5$. C'est dire que, pour $S=x$, l'équilibre est immédiatement atteint.

Pour les valeurs de $o<S<\mathrm{I}$, d'après la disposition des portions de courbes de la famille (fig. I), on voit que l'intersection de $u_{n}=f\left(u_{n-1}\right)$ (équation 2) avec la première bissectrice se fait sous un angle qui va en se réduisant régulièrement de $S=$ I à $S=0$. Par conséquent l'équilibre sera de plus en plus lentement atteint à mesure que $S$ se rapprochera de la valeur zéro. En étudiant la vitesse avec laquelle on atteint la valeur d'équilibre pour $S=0$ on aura donc une limite inférieure de cette vitesse.

$$
\text { Pour } S=0 \quad u_{n}=\frac{u_{n-1}+0,5}{u_{n-1}+\mathrm{I}} \quad \text { et } \quad u_{e}=0,707
$$

et on a successivement, quel que soit $u_{0}: u_{1}=0,5 ; u_{2}=0,666 ; u_{3}=0,700$; $u_{4}=0,706$.

C'est-à-dire qu'en troisième génération la fréquence génique s'écarte en valeur relative de I p. Ioo de la valeur d'équilibre et de I p. I ooo en quatrième génération. Dans tous les cas donc l'équilibre est rapidement atteint. 


\section{II. - ESTIMATION DE LA FRÉQUENCE $u_{e}$ DU GÈNE $P$ A L'ÉQUILIBRE DANS PLUSIEURS POPULATIONS CAPRINES}

\section{A. - Matériel et méthodes}

Venant de deux pays (la Suisse et l'Allemagne) où le maintien de races sans cornes a toujours été un but d'élevage, nous avons de nombreuses données rassemblées par BuEchI (1957) et Brandsch (1959) qui nous donnent des dénombrements à la naissance des intersexués, des femelles et des mâles cornus et mottes dans des populations où le modèle est suivi depuis si longtemps que l'on peut penser que l'équilibre est atteint.

Sachant que la fréquence gamétique de $P$ chez les femelles est toujours égale à 0,5 , les fréquences des différentes catégories à la naissance sont fonction de la seule fréquence gamétique mâle $u_{e}$ (cf. tabl. 1). On peut alors imaginer deux méthodes d'estimation de $u_{e}$, en tenant compte soit de son action sur le cornage, soit sur le déterminisme du sexe.

\section{TABIEAU I}

Proportions théoriques des différentes catégories génotypiquement et phénotypiquement détectables à la naissance en fonction de la fréquence gamétique d'équilibre $\mathbf{u}_{\boldsymbol{e}}$ chez les mâles

\begin{tabular}{|c|c|c|c|}
\hline $\begin{array}{l}\text { Sexe } \\
\text { génétique }\end{array}$ & $\begin{array}{c}\text { Génotypes } \\
\text { en } P\end{array}$ & Phénotypes & $\begin{array}{l}\text { Fréquence } \\
\text { à la naissance }\end{array}$ \\
\hline$\underset{\text { (femelles) }}{\mathrm{XX}}$ & $\begin{array}{l}P p \\
P p \\
P P\end{array}$ & $\begin{array}{l}\text { Femelles cornues } \\
\text { Femelles sans cornes } \\
\text { Intersexués et } \\
\text { pseudo-mâles }\end{array}$ & $\begin{array}{c}\text { I- } u_{e} \\
\frac{1}{4} \\
\frac{u_{e}}{4}\end{array}$ \\
\hline $\begin{array}{c}\text { XY } \\
\text { (mâles) }\end{array}$ & $\begin{array}{l}P p \\
P p \\
P P\end{array}$ & $\begin{array}{l}\text { Mâles cornus } \\
\text { Mâles sans cornes }\end{array}$ & $\begin{array}{l}\frac{\mathrm{I}-u_{e}}{4} \\
\frac{u_{e}+\mathrm{I}}{4}\end{array}$ \\
\hline $\begin{array}{c}\mathrm{XX} \\
\text { (femelles) } \\
\text { XX et XY } \\
\text { (femelles et mâles) }\end{array}$ & $\begin{array}{c}P p \text { ou } p p \\
p p\end{array}$ & $\begin{array}{l}\text { Femelles vraies } \\
\text { Animaux cornus }\end{array}$ & $\begin{array}{l}\frac{2-u_{e}}{4} \\
\frac{\mathrm{I}-u_{e}}{2}\end{array}$ \\
\hline
\end{tabular}

La méthode basée sur le comptage des animaux cornus serait possible en Allemagne, à partir des données de Brandsch (1959), qui est le seul à fournir la fréquence des animaux cornus à la naissance. Mais les chiffres observés sont très en dessous des limites inférieures prévues et en discordance avec les fréquences déduites par d'autres méthodes $(8,15 \%$ pour un minimum de $16,6 \%$ ). Cela tient sans doute du fait que le cornage n'est pas détecté avec sûreté chez tous les animaux qui le présentent, ni à la naissance ni même un peu après, comme le montrent RIcoRDEAU et Bouillon (1969). 


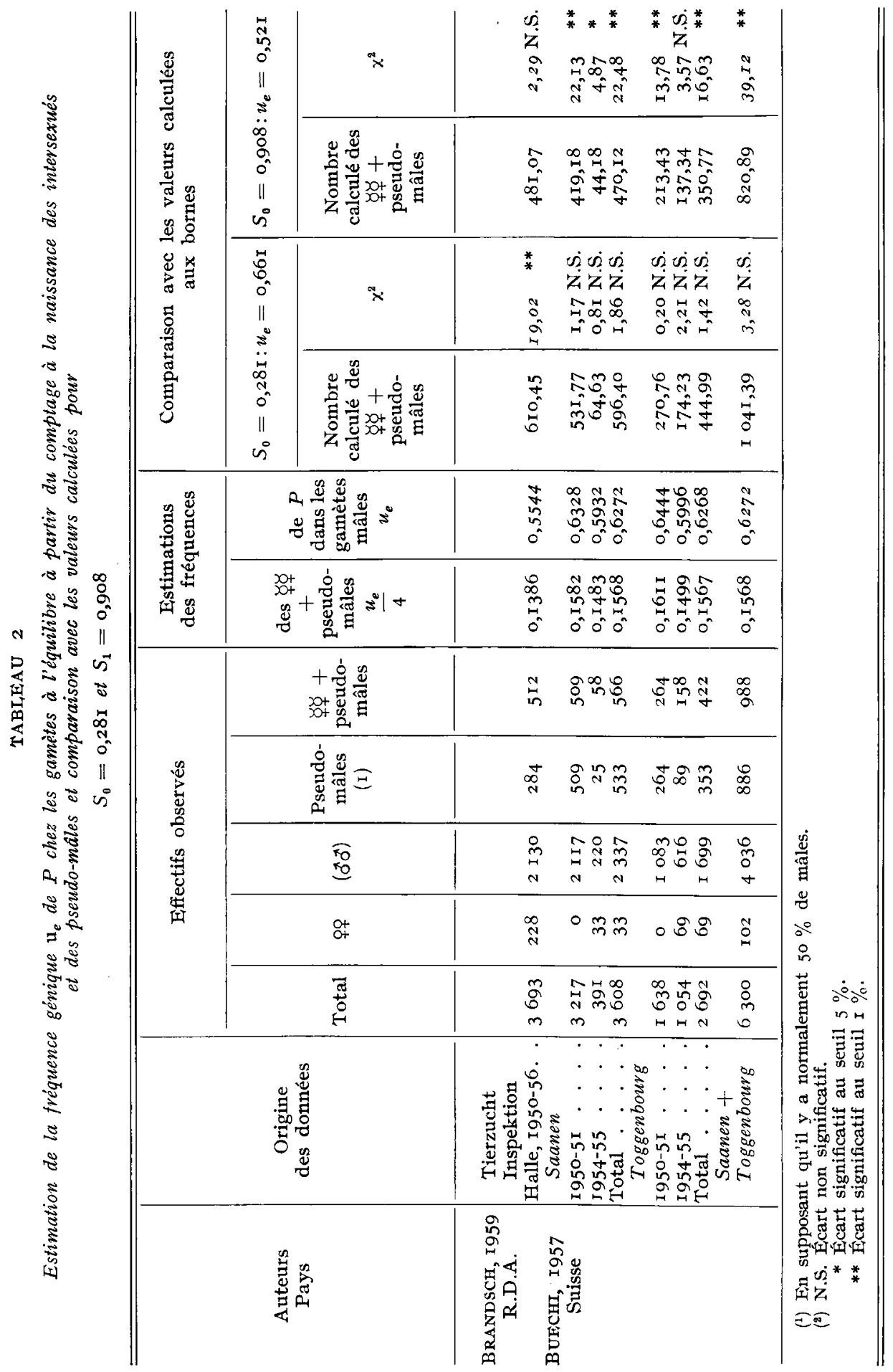


Nous avons donc seulement mis en œuvre la seconde méthode basée sur la fréquence des intersexués et des pseudo-mâles, la plus précise des statistiques que l'on obtient à la naissance. Cette fréquence est le complément à 0,5 de la fréquence des femelles observées à la naissance $\left(^{1}\right)$. Flle est égale au quart de la fréquence génique gamétique mâle (cf. tabl. 1).

\section{B. - Résultats}

L'analyse des données suisses et allemandes est donnée dans le tableau 2 où on les compare aux bornes de l'intervalle théorique de variation.

On a complété cette analyse par une série de tests afin de déterminer si les fréquences observées en Suisse étaient significativement différentes entre elles et avec la valeur établie en Allemagne. Ces tests sont basés sur la méthode proposée par MAURICE et CHARTIER (r954) qui consiste à mesurer l'écart entre les 2 fréquences en terme d'écart-type de lui-même. L'intervalle d'acceptation à 0,95 de l'hypothèse nulle est alors $t=2$ (tabl. 3).

\section{TABLEAU 3}

Comparaison des estimées de la fréquence gamétique mâle du gène $\mathbf{P}$ à l'équilibre en Suisse et en Allemagne en utilisant les fréquences des males et des intersexués à la naissance

\begin{tabular}{|c|c|c|c|}
\hline $\begin{array}{l}\text { Races ou populations } \\
\text { mises en comparaison }\end{array}$ & $n_{1}$ & $n_{2}$ & $t\left({ }^{1}\right)$ \\
\hline 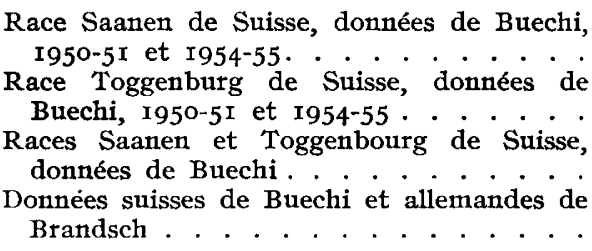 & $\begin{array}{r}3217 \\
1638 \\
3608\end{array}$ & $\begin{array}{r}391 \\
1054 \\
2692 \\
3693\end{array}$ & $\begin{array}{l}\text { 0,513 N.S. } \\
\text { o,794 N.S. } \\
\text { o,01 I N.S. } \\
2,46^{*}\end{array}$ \\
\hline
\end{tabular}

(1) N.S. Écart significatif.

* Écart significatif au seuil $5 \%$.

\section{C. - Discussion}

Comme les données suisses ne sont pas significativement différentes entre elles (tab1. 2) on peut les grouper, ce qui simplifiera la discussion.

On remarque tout d'abord que les deux estimées de $u_{e}$ en Allemagne $(0,5544)$ et en Suisse $(0,6444)$ se placent dans l'intervalle théorique assez restreint que nous avons établi au début de ce mémoire $(0,52 \mathrm{I}-0,66 \mathrm{I})$. Cela confirme l'exactitude de l'hypothèse générale et laisse à penser que les estimées de $b$ et $\beta_{2}$ (cf. LAUvERGNE, I969) se rapprochent assez de la réalité des faits.

Comme la valeur observée en Allemagne ne diffère pas significativement de la borne inférieure, cela signifierait que, dans ce pays, les boucs atteints de sténose

(1) L,e sex-ratio normal de la chèvre exprimé en rapport des mâles sur le nombre total est de 50 p. Ioo, Betrini (1968). Elementi di demografia zootecnica. Produzione animale, Portici, Napoli. 
partielle et donc possiblement fertiles sont tous écartés de la reproduction. D'après ScHöNHERR (I954) cela ne correspond qu'en partie à la réalité car, si les animaux considérés par cet auteur comme inacceptables sont immédiatement castrés,ce ux de la catégorie " acceptables sous réserves " ne sont pas forcément éliminés. Un certain nombre se révèlent cependant stériles et l'on doit, en outre, noter qu'avec l'étiquette obligatoire d' "acceptables sous réserve " dans les transactions commerciales, ils contribuent assez peu à la génération suivante. Ainsi, la valeur de la pénétrance du phénomène parmi les homozygotes $\left(0,9 I_{4}\right)$ correspondrait à la réalité des faits observés en Allemagne.

La situation est différente en Suisse où la fréquence ne s'écarte pas significativement de la fréquence calculée dans le cas où les animaux atteints partiellement et, partant, fertiles sont utilisés. Cela correspond selon WEBER (I969) aux conditions d'élevage suisse et 1'on vérifierait ainsi la valeur de l'expressivité calculée d'après SchöNHERR (I956). Or, si l'on regarde de plus près le travail de cet auteur on se rend compte qu'il a compté parmi les mâles " inacceptables » des animaux à la fois atteints bilatéralement et unilatéralement (ces derniers pouvant être fertiles selon WEBER, r969) et que, par ailleurs il s'aperçoit que, chez les mâles " acceptables sous conditions " un certain nombre sont, en fait stériles. Il faudrait donc admettre que, les erreurs faites dans une catégorie sont compensées par celles faites dans l'autre. Remarquons enfin que cet auteur compte parmi les boucs " acceptables sous conditions " des animaux à testicules hypoplasiques dont on n'avait pas le nombre dans le tableau général des données à partir desquelles on a déterminé les paramètres $b$ et $\beta_{2}$, ce qui nous avait empêché de les déduire. On commettrait ainsi une erreur par excès et pour $b$ et pour $\beta_{1}\left(\beta_{1}=\mathrm{I}-\beta_{2}\right)$. En réalité cela n'est pas si sûr car certains auteurs (CORTEEL et al., I969) ont montré que les animaux sténosiques présentent parfois une hypoplasie marquée des testicules. En outre, il est peu probable que des animaux vraiment hypoplasiques (et donc de sexe chromosomique femelle) aient pu passer avec succès les tests de qualité du sperme pour être classés dans la catégorie d'“ acceptables sous condition ».

Finalement donc, telles qu'elles sont tirées de SchöNHERR qui, rappelons-le malgré leur insuffisance, sont les plus nombreuses dont on pouvait disposer, les estimées de $b$ et $\beta_{2}$ semblent acceptables. Il serait bon toutefois de les obtenir également par d'autres voies, ne serait-ce que parce que, dans le calcul donnant $b$ et $\beta_{2}$, on a utilisé (LAUVERGNE, I969) un résultat de fréquence génique tiré de BRANDSCH (I959), fréquence que l'on compare dans le présent article à la valeur théorique sans tenir compte de ce fait dans les comparaisons statistiques à cause de l'énorme complication que cela introduirait dans le calcul des équations estimatrices.

\section{CONCLUSION : LES CONSÉQUENCES POUR L'ÉLEVAGE CAPRIN D'UN TEL SCHÉMA DE SÉLECTION}

Dans le schéma de sélection envisagé, on a vu déjà que, quel que soit $S$, la moitié des femelles sont écartées de la reproduction, qu'elles soient $P P$ (intersexuées, mâles stériles ou femelles stériles) ou $p p$ (cornues). 
Chez les mâles, lorsque l'équilibre est atteint, la proportion $m$ des individus écartés de la reproduction à cause des effets du gène est une fonction de $u_{e}$ qui prend la forme :

$$
m=0,5\left[\mathrm{I}+\frac{0,5-u_{e}}{r\left(\mathrm{I}-u_{e}\right)}\right]
$$

$u_{e}$ variant entre $0,52 \mathrm{I}$ et $0,66 \mathrm{I}, m$ variera entre 0,479 et 0,279 (cf. fig. 3 ).

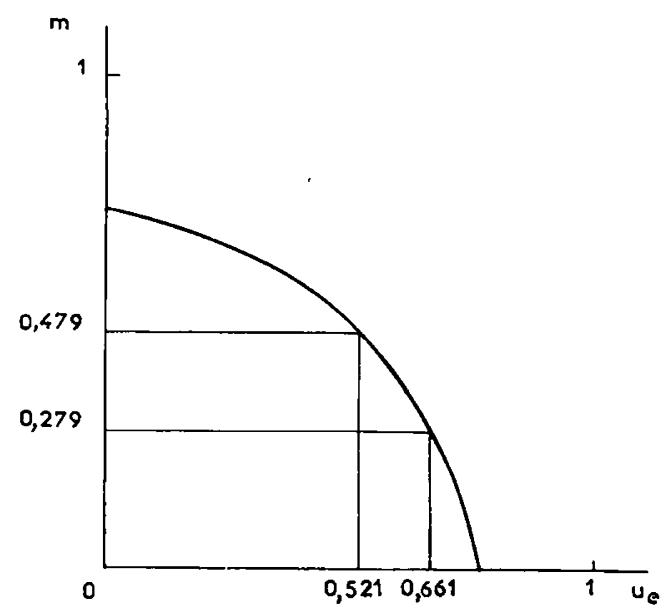

IIG. 3. - Proportion $\mathrm{m}$ des mâles qui doivent être éliminés en fonction de $\mathrm{u}_{e}$ fréquence à l'équilibre de $P$ dans les gamètes mâles.

Un tel handicap sélectif (50\% des femelles, 28 à $48 \%$ des mâles éliminés) n'est guère supportable dans les perspectives d'une sélection rationnelle de la chèvre avec contrôle des performances et testage des boucs. A cet inconvénient qui ne serait peut-être pas très fort aux yeux des éleveurs traditionnels si les divers animaux pouvaient tous être détectés à la naissance, s'ajoutent les pertes dues à la détection tardive des mâles stériles. C'est cet aspect qu'ont retenu surtout les auteurs allemands comme ScHöNHERR (r954) qui chiffre le manque à gagner sur le bouc qu'il a fallu élever jusqu'à l'âge de la reproduction et qui ne pourra être vendu comme reproducteur, le manque à gagner sur les chèvres qui, saillies par des boucs stériles resteront vides, sans compter les frais occasionnés par le contrôle systématique de tous les jeunes reproducteurs.

Dans ces conditions, il ne semble guère sage de chercher à utiliser la surprolificité des mâles $P P$ car cela conduirait à produire des animaux homozygotes. I1 est possible, par contre, d'utiliser la surprolificité des femelles hétérozygotes, au moins dans la période de transition précédant la disparition totale du gène $P$ dans les élevages laitiers. 


\section{SUMMARY \\ FREQUENCY AT EQUILIBRIUM OF THE GENE OF AIPINE ORIGIN RESPONSIBLE FOR POLLEDNESS AND INTERSEXUALITY IN SOME POPULATIONS OF GOATS}

Considering all the behavioral aspects of the autosomal $P$ gene responsable for absence of horns and intersexuality in the domestic goat, the frequency at equilibrium of this gene (in the model where only animals without horns are retained for reproduction) is always 0.5 in the female gametes. It varies between 0.521 and 0.661 in the male gametes, depending on whether all homozygous $P P$ males presenting partial epididymal disorders are kept, or discarded from reproduction. The gametic male frequencies observed in German and Swiss populations are 0.5544 and 0.6444 , respectively. They are situated in the determined theoretical interval, and cannot be statistically distinguished; that is, the first interval of the lower limit (all rejected $P P$ males having epididymal disorders) the second interval of the upper limit (all $P P$ males having partial epididymites and being admitted to reproduction). This corresponds to about the selection conditions as observed in Germany or Switzerland regarding the above-mentioned males. Consequently, it seems that the proposed theoretical model is valuable. This verifies its various basic hypotheses, and leads us to believe that estimations of different parameters come close to reality.

In order to maintain such a model, one-half of the females and 28-48 per cent of the males in each generation should be eliminated, whether they are horned or have genital malformations causing sterility.

\section{RÉFERENCES BIBLIOGRAPHIQUES}

BRANDSCH H., 1959. Die Vererbung geschlechtlicher Missbildung und des Hornes bei der Hausziege in ihrer gegenseitigen Beziehung. Arch. Gefügelzucht. Kleintierkde, 8, 310-362.

BUECHI H. F., I957. Untersuchungen über das verschobene Geschlechtsverhältnis, die Intersexualität und die Fruchtbarkeit bei der Milchziege. Z. Tierzücht. ZïchtBiol., 69, 30-9o.

IAAUVERGNe J.-J., I969. Progrès des connaissances génétiques sur l'intersexualité associée à l'absence de cornes chez la Chère domestique d'origine alpine. Ann. Génét. Sél. anim., 1, 403-4ra.

RICORDEAU G., 1969. Surprolificité des génotypes sans cornes dans les races caprines alpine Saanen, alpine chamoisée et poitevine. Ann. Génét. Sél. anim., 1, 391-395.

Ricordeau G., Boullon J., 1969. Variations d'âge d'apparition du cornage dans les races caprines alpine Saanen, alpine chamoisée et poitevine. Ann. Génét. Sèl. anim. 1, 397-40r.

RrCordeau G., IAAUVERGNe J.-J., 1967. Hypothèse génétique unique pour expliquer la présence d'intersexués, de mâles en excès et de mâles stériles en race caprine Saanen. Ann. Zootech., 16, 323-334.

SCHÖNHERR S., I954. Die Unfruchtbarkeit der Ziegenböcke, ihre Verbreitung, frühzeitige Errkennung und Bekämpfung. Vet.-med. Diss. Frei Universität, Berlin.

Soller M., LAor M., Barnea R., Wriss Y., Ayalon N., I963. Polleduess and infertility in male Saancn goats. J. Hered., 54, $237-240$. 